\title{
CORRECTION
}

\section{Correction to: The 2019 World Molecular Imaging Congress (WMIC) and Molecular Imaging and Biology (MIB) Awards}

\author{
Jason S. Lewis, ${ }^{1}$ Rosemery Membreno ${ }^{2}$ \\ ${ }^{1}$ Molecular Imaging and Biology \& Memorial Sloan Kettering Cancer Center, New York, NY, USA \\ ${ }^{2}$ Molecular Imaging and Biology \& World Molecular Imaging Society, Culver City, CA, USA
}

Correction to: Mol Imaging Biol

https://doi.org/10.1007/s11307-019-01444-0

In the sixth paragraph:

"...the Commercial Innovation of the Year Award went to Emile Beaulieu of Photon etc. for their work, "Real Time in vivo Imaging of ICG in the NIR II with the IR VIVOTM Imaging System";"

should read:

"...the Commercial Innovation of the Year Award went to Emilie Beaulieu Ouellet of Photon etc. for their work, "Real Time in vivo Imaging of ICG in the NIR II with the IR VIVO Imaging System";".

In the sixth paragraph:

"The winner of the first annual WMIC Science Elevator-modeled after the very successful and popular postdoc slam/grand slam competitions held at many universities - was Candice Asmore-Harris of King's College London;"

should read:

"The winner of the first annual WMIC Science Elevator-modeled after the very successful and popular postdoc slam/grand slam competitions held at many universities - was Candice Ashmore-Harris of King's College London;".

The online version of the original article can be found at https://doi.org/ 10.1007/s11307-019-01444-0 